\title{
A-site doped perovskite oxide strongly interface coupling with carbon nanotubes as a promising bifunctional electrocatalyst for flexible solid-state Zn-air battery
}

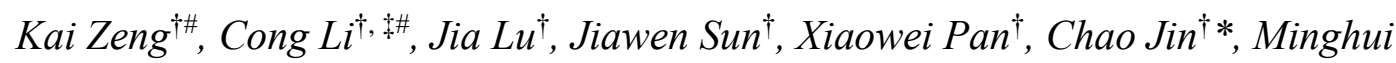

Wei ${ }^{\dagger}$, Ruizhi Yang ${ }^{\dagger *}$

${ }^{\dagger}$ College of Energy, Soochow Institute for Energy and Materials Innovations,

Soochow University, Suzhou 215006, China

\$East China Institute of Photo- Electronic, Suzhou 215163, China

${ }^{\#} \mathrm{~K}$. Zeng and C. Li contributed equally to this work. 


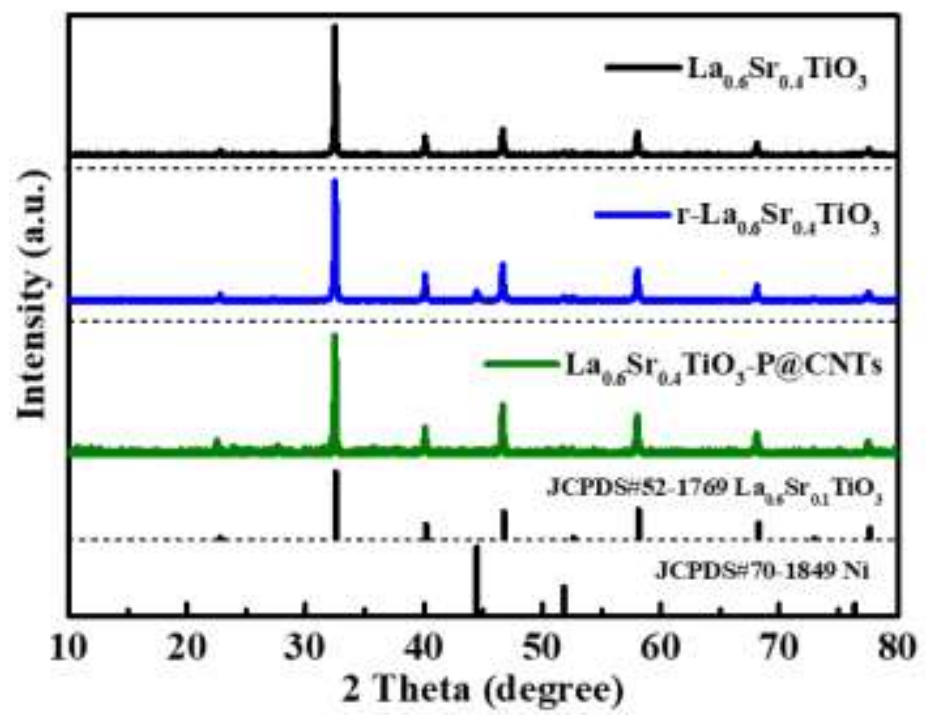

Figure S1. XRD patterns of $\mathrm{La}_{0.6} \mathrm{Sr}_{0.4} \mathrm{TiO}_{3}, \quad r-\mathrm{La}_{0.6} \mathrm{Sr}_{0.4} \mathrm{TiO}_{3}$ and $\mathrm{La}_{0.6} \mathrm{Sr}_{0.4} \mathrm{TiO}_{3}-\mathrm{P} @ \mathrm{CNTs}$, respectively. 

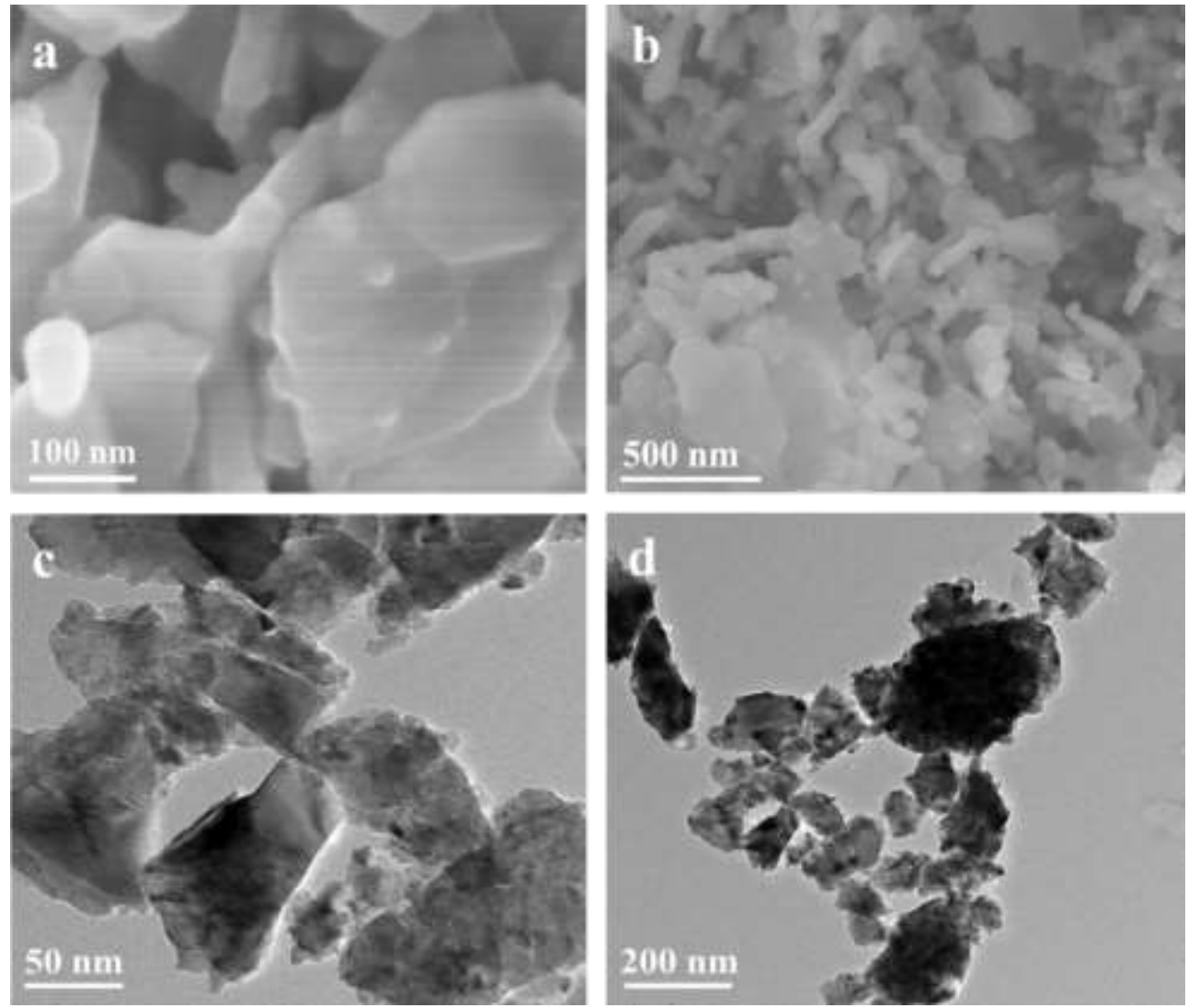

Figure S2. (a, b) SEM images. (c, d) TEM images of $\mathrm{La}_{0.6} \mathrm{Sr}_{0.4} \mathrm{TiO}_{3}$. 


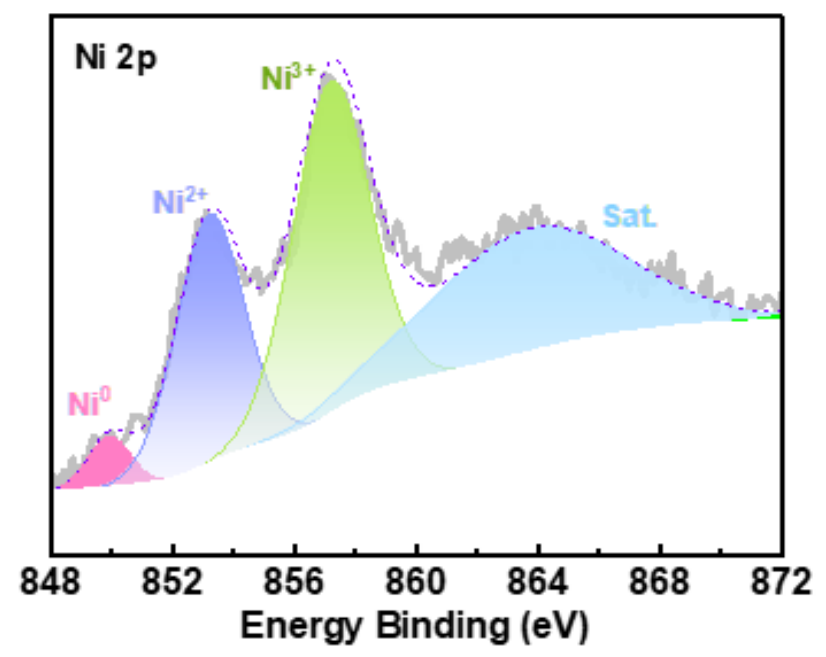

Figure S3. The Ni XPS spectrum of $\mathrm{La}_{0.6} \mathrm{Sr}_{0.4} \mathrm{TiO}_{3}-\mathrm{P} @ \mathrm{CNTs}$. In the Ni XPS spectrum of $\mathrm{La}_{0.6} \mathrm{Sr}_{0.4} \mathrm{TiO}_{3}-\mathrm{P} @ \mathrm{CNTs}$, it exists metallic state, divalent and trivalent of nickel. 

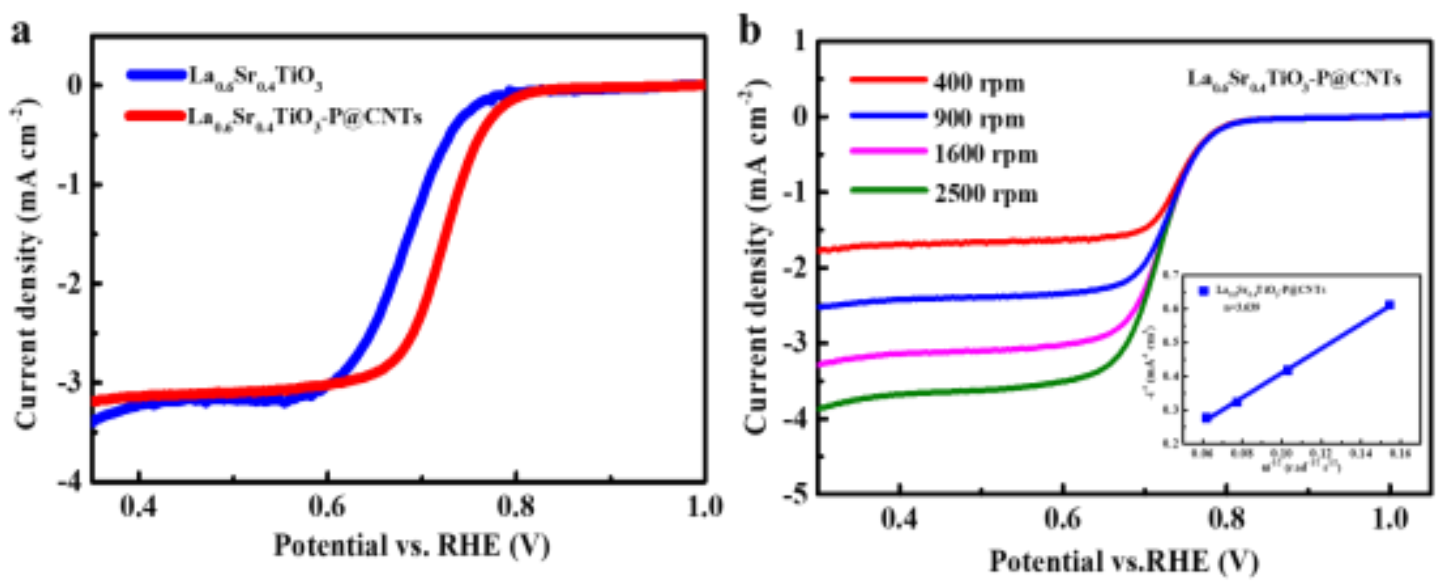

Figure S4. (a) ORR polarization curves of $\mathrm{La}_{0.6} \mathrm{Sr}_{0.4} \mathrm{TiO}_{3}$ and $\mathrm{La}_{0.6} \mathrm{Sr}_{0.4} \mathrm{TiO}_{3}-\mathrm{P} @ \mathrm{CNTs}$ at $1600 \mathrm{rpm}$. (b) ORR polarization curves of $\mathrm{La}_{0.6} \mathrm{Sr}_{0.4} \mathrm{TiO}_{3}-\mathrm{P} @ \mathrm{CNTs}$ with different rotating speeds $(400,900,1600,2500 \mathrm{rpm})$. Inset: calculation of transfer electron number. 

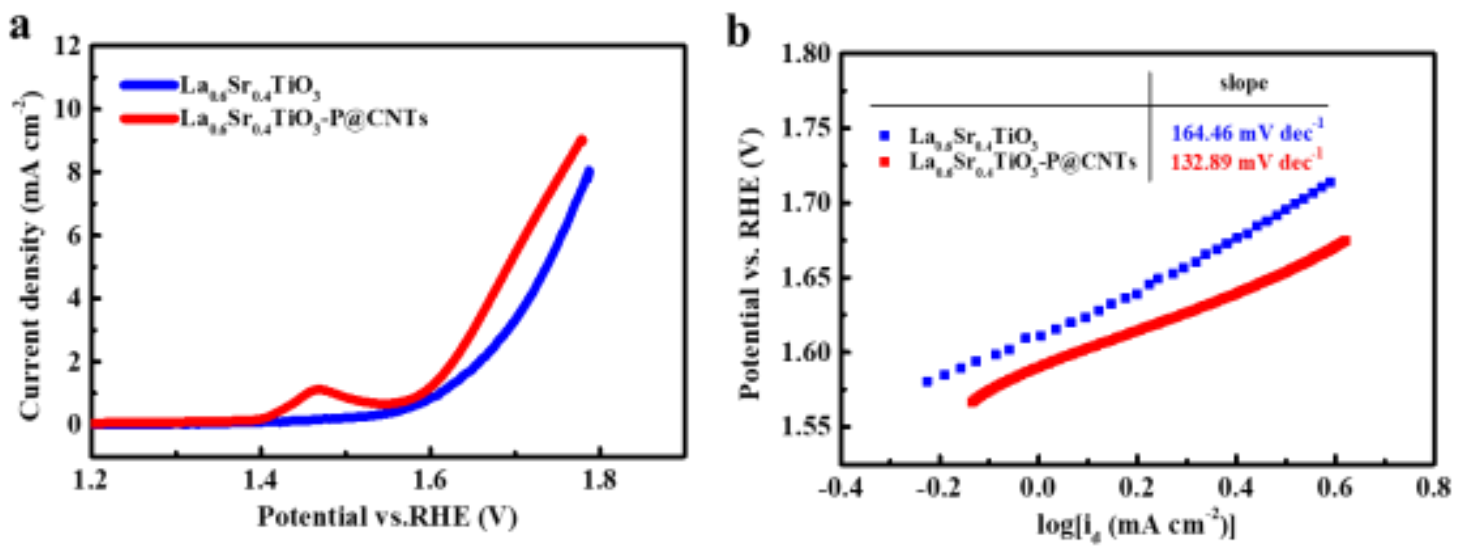

Figure S5. (a) OER polarization curves and (b) Tafel slope of $\mathrm{La}_{0.6} \mathrm{Sr}_{0.4} \mathrm{TiO}_{3}$ and $\mathrm{La}_{0.6} \mathrm{Sr}_{0.4} \mathrm{TiO}_{3}-\mathrm{P} @$ CNTs. 


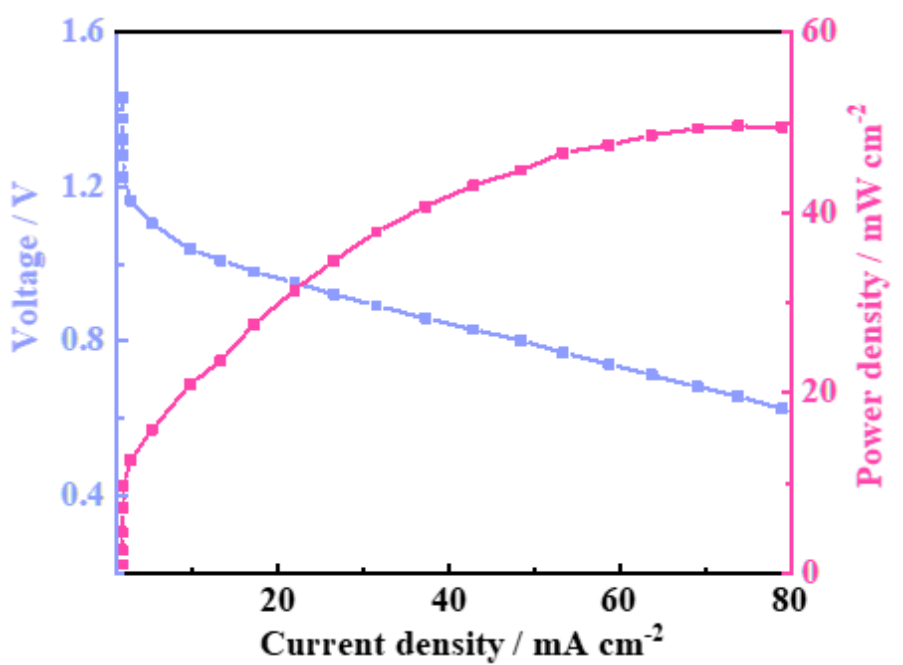

Figure S6. The power density and discharge curves of $\mathrm{Pt} / \mathrm{C}-\mathrm{IrO}_{2} . \mathrm{Pt} / \mathrm{C}-\mathrm{IrO}_{2}$ couple shows a power density of $49 \mathrm{~mW} \mathrm{~cm}$, which is comparable that of $\mathrm{La}_{0.6} \mathrm{Sr}_{0.4} \mathrm{TiO}_{3}-\mathrm{P} @$ CNTs. 


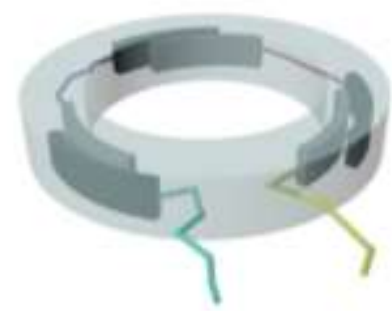

b

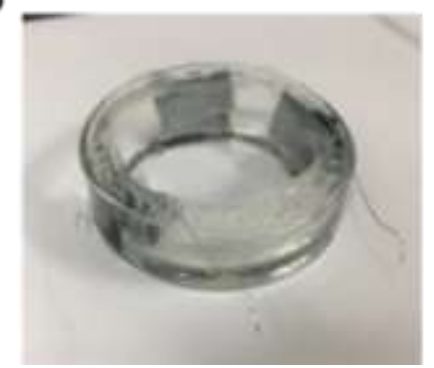

c

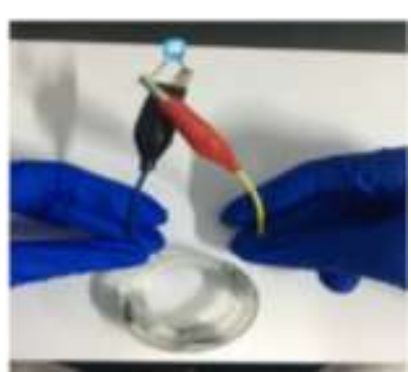

Figure S7. (a, b) Schematic illustration and digital photo of flexible solid Zn-air battery. (c) Digital photo of a colorful LED powered with three series-connected flexible solid $\mathrm{Zn}$-air battery by an integral casting process. 
Table S1. The summary of ORR and OER performance for $\mathrm{La}_{0.6} \mathrm{Sr}_{0.4} \mathrm{TiO}_{3}$, $\mathrm{La}_{0.6} \mathrm{Sr}_{0.4} \mathrm{TiO}_{3}-\mathrm{P} @ \mathrm{CNTs}, \mathrm{Pt} / \mathrm{C}$ and $\mathrm{IrO}_{2}$

\begin{tabular}{cccccc}
\hline Catalyst & $\begin{array}{c}\text { ORR } \\
\mathrm{E}_{\text {onset }} \\
/ \mathrm{V}\end{array}$ & $\begin{array}{c}\text { ORR } \\
\mathrm{E}_{1 / 2} / \mathrm{V}\end{array}$ & $\begin{array}{c}\text { OER } \\
\mathrm{E}_{\text {onset }} \\
/ \mathrm{V}\end{array}$ & $\begin{array}{c}\text { OER Tafel } \\
\text { slope } / \mathrm{mV} \\
\mathrm{dec}^{-1}\end{array}$ & Reference \\
\hline $\mathrm{La}_{0.6} \mathrm{Sr}_{0.4 \mathrm{TiO}_{3}-\mathrm{P} @ \text { CNTs. }}$ & 0.83 & 0.73 & 1.59 & 132.89 & This work \\
$\mathrm{La}_{0.6} \mathrm{Sr}_{0.4} \mathrm{TiO}_{3}$ & 0.78 & 0.68 & 1.65 & 164.46 & This work \\
$\mathrm{IrO}_{2}$ & 0.65 & 0.29 & 1.63 & 86.2 & $\mathrm{~S} 1$ \\
$\mathrm{Pt} / \mathrm{C}$ & 0.97 & 0.9 & 1.66 & 354.9 & $\mathrm{~S} 1$ \\
\hline
\end{tabular}


Table S2. Comparison of Zn-air battery performance for $\mathrm{La}_{0.6} \mathrm{Sr}_{0.4} \mathrm{TiO}_{3}-\mathrm{P} @ \mathrm{CNTs}$ and recent reported materials.

\begin{tabular}{|c|c|c|c|c|c|}
\hline Catalyst & $\begin{array}{l}\text { Voc } \\
/ \mathrm{V}\end{array}$ & $\begin{array}{l}\text { Power density } \\
\qquad / \mathrm{W} \mathrm{cm}^{-2}\end{array}$ & $\begin{array}{c}\text { Aqueous } \\
\text { /Solid-state } \\
\text { (ZAB) }\end{array}$ & $\begin{array}{l}\text { Cycling } \\
\text { time } / \mathrm{h}\end{array}$ & Reference \\
\hline $\begin{array}{c}\mathrm{La}_{0.6} \mathrm{Sr}_{0.4} \mathrm{TiO}_{3}-\mathrm{P} @ \mathrm{C} \\
\mathrm{NTs} .\end{array}$ & 1.47 & 35 & Solid-state & 31 & This work \\
\hline $\mathrm{Ce}-\mathrm{LaCoO}_{3}$ & 1.32 & 31 & Solid-state & 8 & S2 \\
\hline $\mathrm{CaMnO}_{3}$ & $\sim 1.41$ & 25 & Solid-state & -- & S3 \\
\hline $\mathrm{D}-\mathrm{CaMnO}_{3}$ & 1.46 & 149 & Solid-state & 34 & S3 \\
\hline NO-G@CP-2 & 1.33 & 65.1 & Solid-state & 18 & S4 \\
\hline $\begin{array}{c}\mathrm{La}_{0.7} \mathrm{Sr}_{0.3} \mathrm{Co}_{0.9} \mathrm{Pd}_{0.03} \\
\mathrm{O}_{2.85}\end{array}$ & 1.5 & 52 & Solid-state & 60 & S5 \\
\hline $\mathrm{S}-\mathrm{LaCoO}_{3}$ & 1.38 & -- & Solid-state & 8 & S6 \\
\hline $\mathrm{FeP} / \mathrm{Fe}_{2} \mathrm{O}_{3} @ \mathrm{NPCA}$ & 1.42 & 40.8 & Solid-state & 8.3 & S7 \\
\hline $\mathrm{La}_{0.9} \mathrm{Ce}_{0.1} \mathrm{MnO}_{3}$ & -- & 124.5 & Aqueous & 28 & S8 \\
\hline $\begin{array}{c}\mathrm{Ba}_{0.6} \mathrm{Sr}_{0.4} \mathrm{Co}_{0.79} \mathrm{Fe}_{0.21} \\
\mathrm{O}_{2.67} / \mathrm{NiFe} \mathrm{LDH}\end{array}$ & 1.4 & 52.8 & Aqueous & 100 & S9 \\
\hline $\mathrm{LaNiO}_{3} @ \mathrm{FeOOH}$ & 1.43 & -- & Aqueous & 16.7 & $\mathrm{~S} 10$ \\
\hline
\end{tabular}




\section{Reference:}

(S1) Aijaz A.; Masa J.; Rçsler C.; Xia W.; Weide P.; Botz A. J. R.; Fischer R. A.; Schuhmann W.; Muhler. M. Co@ $\mathrm{Co}_{3} \mathrm{O}_{4}$ Encapsulated in Carbon Nanotube-Grafted Nitrogen-Doped Carbon Polyhedra as an Advanced Bifunctional Oxygen Electrode. Angew. Chem. Int. Ed. 2016, 55, 4087-4091.

(S2) Qian J. M.; Wang T. T.; Zhang Z. M.; Liu Y. G.; Li J. F.; Gao D. Q. Engineered spin state in $\mathrm{Ce}$ doped $\mathrm{LaCoO} 3$ with enhanced electrocatalytic activity for rechargeable Zn-Air batteries. Nano Energy 2020, 74, 104948.

(S3) Yang F.; Xie J. H.; Rao D. W.; Liu X. Q.; Jiang J. X.; Lu X. H. Octahedral distortion enhances exceptional oxygen catalytic activity of calcium manganite for advanced Zn-Air batteries. Nano Energy 2021, 85, 106020.

(S4) Zheng X. J.; Cao X. C.; Zeng K.; Sun Z. H.; Yan J.; Li X. W.; Jin C.; Chen X.; Yang R. Z. Cotton pad-derived large-area 3D N-doped graphene-like full carbon cathode with an O-rich functional group for flexible all solid $\mathrm{Zn}$-air batteries. J. Mater. Chem. A, 2020, 8, 11202-11209.

(S5) Majee R.; Das T.; Chakraborty S.; Bhattacharyya S. Shaping a Doped Perovskite Oxide with Measured Grain Boundary Defects to Catalyze Bifunctional Oxygen Activation for a Rechargeable Zn-Air Battery. ACS Appl. Mater. Interfaces 2020, 12, 40355-40363.

(S6) Ran J.; Wang T.; Zhang J.; Liu Y.; Xu C.; Xi S.; Gao D. Modulation of Electronics of Oxide Perovskites by Sulfur Doping for Electrocatalysis in Rechargeable Zn-Air Batteries. Chem. Mater. 2020, 32, 3439-3446.

(S7) Wu K.; Zhang L.; Yuan Y.; Zhong L.; Chen Z.; Chi X.; Lu H.; Chen Z.; Zou R.; Li T.; Jiang C.; Chen Y.; Peng X.; Lu J. An Iron-Decorated Carbon Aerogel for Rechargeable Flow and Flexible Zn-Air Batteries. Adv. Mater. 2020, 32, 2002292.

(S8) Zhang Y. D.; Feng F. X.; Zhang C. H.; Zheng Q. L.; Wang C. C.; Hu H. B.; Wu M. Z.; Guo Y. M. Enhanced Catalytic Activity of $\mathrm{LaMnO}_{3}$ by A-Site Substitution as Air Electrode of Zn-Air Batteries with Attractive Durability. Energy Fuels 2020, 34, 10170-10177.

(S9) Majee R.; Islam Q. A.; Bhattacharyya S. Surface Charge Modulation of Perovskite Oxides at the Crystalline Junction with Layered Double Hydroxide for a Durable Rechargeable Zinc-Air Battery. ACS Appl. Mater. Interfaces 2019, 11, 35853-35862.

(S10) Li Z. S.; Lv L.; Ao X.; Li J.-G.; Sun H. C.; An P. D.; Xue X. Y.; Lie Y.; Liu M.; Wang C. D.; Liu. M. L. An effective method for enhancing oxygen evolution kinetics of $\mathrm{LaMO}_{3}(\mathrm{M}=\mathrm{Ni}, \mathrm{Co}, \mathrm{Mn})$ perovskite catalysts and its application to a rechargeable zinc-air battery. Appl. Catal. B: Environ. 2020, 262, 118291. 